\title{
Effects of Dog-Assisted Therapy on the Physical Function and Communication Skills of Adults with Autism: A Study Protocol for a Controlled Study
}

\author{
Isabel Gómez-Calcerrada ${ }^{1}$, Ana Myriam Lavín-Pérez ${ }^{2, *}{ }^{\mathbb{D}}$, Santos Villafaina ${ }^{3}{ }^{\mathbb{D}}$, Juan Carlos Rueda-Rubio ${ }^{4}$, \\ Beatriz Rivera-Martín ${ }^{5,6}{ }^{\circ}$, Israel González-García ${ }^{5}$, Eugenio Merellano-Navarro ${ }^{7, *}$ (D) Marta Mateo-Garitagoitia ${ }^{8}$, \\ Carlota González-Plaza ${ }^{9}$ and Daniel Collado-Mateo ${ }^{2,5}{ }^{\mathbb{B}}$
}

1 Department of Occupational Therapy, Espacio Terapéutico Gaia, 28770 Madrid, Spain; t.ocupacional.isabel@gmail.com

2 Centre for Sport Studies, Rey Juan Carlos University, Fuenlabrada, 28943 Madrid, Spain; daniel.collado@urjc.es

3 Physical Activity and Quality of Life Research Group (AFYCAV), Faculty of Sport Sciences, University of Extremadura, 10003 Caceres, Spain; svillafaina@unex.es

4 Occupational Therapy Department, Asociación de Padres de Niños con Autismo (APNA), 28708 Madrid, Spain; terapiaocupacional.ramib@apna.es

check for

updates

Citation: Gómez-Calcerrada, I.; Lavín-Pérez, A.M.; Villafaina, S.;

Rueda-Rubio, J.C.; Rivera-Martín, B.; González-García, I.;

Merellano-Navarro, E. Mateo-Garitagoitia, M.; González-Plaza, C.; Collado-Mateo, D. Effects of Dog-Assisted Therapy on the Physical Function and Communication Skills of Adults with Autism: A Study Protocol for a Controlled Study. Appl. Sci. 2021, 11, 10650. https://doi.org/10.3390/ app112210650

Academic Editor: Qi-Huang Zheng

Received: 9 September 2021

Accepted: 8 November 2021

Published: 12 November 2021

Publisher's Note: MDPI stays neutral with regard to jurisdictional claims in published maps and institutional affiliations.

Copyright: (c) 2021 by the authors. Licensee MDPI, Basel, Switzerland. This article is an open access article distributed under the terms and conditions of the Creative Commons Attribution (CC BY) license (https:/ / creativecommons.org/licenses/by/ $4.0 /)$.
5 Animal-Assisted Intervention Office, Rey Juan Carlos University, Móstoles, 28933 Madrid, Spain; beatriz.rivera@urjc.es (B.R.-M.); oficina.iaa@urjc.es (I.G.-G.)

6 Department of Communication and Sociology Sciences, King Juan Carlos University, Fuenlabrada, 28943 Madrid, Spain

7 EFISAL Investigation Group, Universidad Autónoma de Chile, Talca 3460000, Chile

8 Social Action Area, Autismo Madrid Federation, 28029 Madrid, Spain; asesoramiento@autismomadrid.es

9 Animal Nature Association, 28232, Madrid, Spain; funcionaldiversidad@hotmail.com

* Correspondence: am.lavin.2018@alumnos.urjc.es (A.M.L.-P.); emerellano@gmail.com (E.M.-N.)

\begin{abstract}
Background and Objectives: Despite the scientific evidence of the positive effects of animalassisted therapy (AAT) in adults with autism spectrum disorder (ASD), it is not still considered an evidence-based practice for this population. The proposed study will aim to evaluate the effects of a dog-assisted therapy program on gait, posture, and communication skills. Materials and Methods: A total of 24 adults with ASD will participate in this non-randomized controlled trial. The experimental group will involve 12 participants who will receive AAT for 10 weeks, at two sessions per week. The focus of these sessions will be conducting different activities and physical exercises, facilitated by dogs. The control group will receive treatment as usual. The outcome measures will be the ability to walk and climbing stairs, balance, risk of falls and communication skills. Conclusions: The role of dogs as facilitators of the movement may lead to relevant benefits in the gait, posture and communication skills of adults with ASD, improving their ability to perform activities of daily living.
\end{abstract}

Keywords: animal-assisted therapy; autism spectrum disorder; dog-assisted therapy; rehabilitation

\section{Introduction}

Autism spectrum disorder (ASD) is a range of alterations of neurobiological origin that compromises typical human development. Patients with ASD are commonly characterized, according to the DSM-5 [1], by permanent deficits in communication and social interaction, repetitive and restricted patterns of behavior, activities, and interests, which interfere in their daily functioning [2]. Although the most studied ASD impairments are social and cognitive deficits, different alterations to gait, which is an essential part of carrying out the activities of daily living (ADLs), have recently been identified, as compared with control groups without ASD [3,4]. Concurrently, reductions in cadence, stride width, gait cycle length, and in the capacity to attenuate the oscillations from the trunk to the head have been observed, in contrast with healthy controls $[3,4]$. 
Regarding therapeutic approaches, behavioral and individualized psychosocial treatments are the most frequent interventions due to the strong empirical evidence of their efficacy [5]. Although the effects of drugs are limited, they can be employed as an adjunct to non-pharmacological therapies. Currently, some evidence-based non-pharmacological practices to treat ASD symptoms are background-based interventions (ABI), augmentative and alternative communication (AAC), cognitive-behavioral strategies, differential reinforcement of alternative, incompatible or other behavior, and functional behavior evaluation, among others [6,7].

Moreover, a new therapy, animal-assisted therapy (AAT), is recently gaining popularity for people with ASD because it increases patients' physical involvement through physical or psychosocial tasks with dogs, horses, dolphins, etc. [7]. AAT, a type of animalassisted intervention (AAI), is a goal-oriented, planned, and structured therapeutic intervention, directed by qualified professionals, and focused on the development of physical functioning and cognitive, behavioral, and/or socio-emotional patients' $[8,9]$. The scientific literature supporting AAT points out that this interaction with an animal could benefit users, leading to calming effects, reductions of loneliness and depression, increases in socializing and motivational effects [10]. AAT has been widely developed with ASD patients, demonstrating increases in social interaction and communication, together with reductions in behavior problems, stress, and the severity of ASD side-effects [11]. A recent study found improvements in patients' anxiety and self-esteem and showed that participants in AAT programs find it applicable and relevant because the positive results obtained by way of relaxation, fun, or self-knowledge, and the dog-contact experience itself [12]. One remarkable aspect of AAT is the positive valuation by health personnel $[13,14]$ and the high rates of adherence, which could be related to patients' positive perception of such experiences [15].

In order to explore the previous studies conducted in the field of ASD and dogassisted therapy, a literature review was performed. It was conducted on the well-known database PubMed. The search string used was: "(Autism) AND (dog or canine)". A total of 130 articles were retrieved on October 10, 2021 (date of the search). Of the 130 articles, 12 were concerned on the effect of dog-assisted therapies in people with ASD, focusing on social [15-18], behavioral aspects [19-24] or physiological variables, such as cortisol levels $[25,26]$. These articles were published between 2010 and 2021. Furthermore, 9 of the 13 articles were focused on children with ASD, which agrees with the conclusions of a previous systematic review [27]. As noted in that literature review, no prior studies have examined the effects of a dog-assisted therapy program on the main outcome measures of walking performance, balance or the risk of falling. This would be relevant due to the impact of ASD on adults physical function [3,4]. Furthermore, it would also be interesting to asses communication skills. Thus, the main aim of the current study is to evaluate the effects of a dog-assisted therapy program on the physical function and communication skills of adults with ASD. The specific objectives are to analyze the effects of AAT in patients' gait, ability to go up and downstairs, balance, communicative intention and social interaction.

Given the mentioned potential benefits of AAT in people with ASD, it is expected that the presented program would lead to improvements in physical functionality and to a consequent reduction in the risk of falls in adults with ASD. Similarly, it has been hypothesized that this program will be able to improve the communication skills and social interactions of this group.

\section{Materials and Methods}

\subsection{Study Design}

The proposed study is a non-randomized controlled clinical trial, which will be carried out in parallel in two different centers (the non-randomization design reasons are explained below). Each location will involve 12 patients, with 6 belonging to a control group and 6 to the experimental group. Therefore, the total sample will be 24 adults with ASD ( 12 in 
the control group and 12 in the experimental group). The study methods described below follow the SPIRIT 2013 Statements Items to correctly fulfill the standard protocol items for Critical Trials [28]. As for having a clear comprehension of the design, in Table 1 represents the schedule regarding the SPIRIT guidelines.

Table 1. Study schedule following SPIRIT guideline.

\begin{tabular}{|c|c|c|c|c|c|c|c|}
\hline \multicolumn{8}{|c|}{ STUDY PERIOD } \\
\hline \multirow[b]{2}{*}{ TIMEPOINT } & \multirow{2}{*}{$\begin{array}{c}\text { Enrolment } \\
-t_{1}\end{array}$} & \multirow{2}{*}{$\begin{array}{c}\text { Allocation } \\
0\end{array}$} & \multicolumn{4}{|c|}{ Post-Allocation } & \multirow{2}{*}{$\frac{\text { Close-Out }}{t_{x}}$} \\
\hline & & & $t_{1}$ & $t_{2}$ & $t_{3}$ & $\mathrm{t}_{4}$ & \\
\hline \multicolumn{8}{|l|}{ ENROLMENT: } \\
\hline Eligibility screen & $x$ & & & & & & \\
\hline Informed consent & $x$ & & & & & & \\
\hline Blinding and confidentiality & $x$ & & & & & & \\
\hline Allocation & & $x$ & & & & & \\
\hline \multicolumn{8}{|l|}{ INTERVENTIONS: } \\
\hline \multicolumn{8}{|l|}{$\begin{array}{l}\text { Animal Assisted Therapy } \\
\text { Group }\end{array}$} \\
\hline Control Group & \multicolumn{7}{|c|}{$\longmapsto$} \\
\hline \multicolumn{8}{|l|}{ ASSESSMENTS: } \\
\hline Sociodemographic variables & $x$ & & & & & & \\
\hline $\begin{array}{l}\text { Walking performance, balance } \\
\text { and risk of falls, and ability to } \\
\text { climb and descend stairs }\end{array}$ & $x$ & & & & & $x$ & \\
\hline Communication skills & $x$ & & & $x$ & & $\mathrm{X}$ & \\
\hline Analysis of results & $x$ & & & & & & $x$ \\
\hline Result comparison & & & & & & & $x$ \\
\hline
\end{tabular}

$\mathrm{t}_{1}$ : 1st-4th week; $\mathrm{t}_{2}$ : 5 th week; $\mathrm{t}_{3}$ : 6th-10th week; $\mathrm{t}_{4}$ : 11 th week.

\subsection{Ethical Approval and Registration}

The study has been approved by the ethical committee of the Rey Juan Carlos University (approval number: 1901202103121) and the University of Extremadura (register identification number: 42//2021). After the positive response of the ethics committees, the trial was registered at ClinicalTrials.gov, recognized by the World Health Organization (WHO) and the International Committee of Medical Journal Editors (ICMJE), under the identification number of NCT04859998. Both the study registration and the positive ethics committee approval were obtained recruitment, whereas the current protocol was written after this process had completed.

Regarding consistency in the informed consent process, all participants or their legal guardians will be informed about all the relevant aspects of this study before starting their program. Accordingly, all will sign the informed consent before being included in the study.

\subsection{Participants}

A total of 24 adults with ASD will participate in the current study belonging twelve to the intervention group $(n=12)$ and twelve to the control group $(n=12)$. The study will be conducted in the center and facilities where they are usually living. The inclusion and exclusion criteria to which they were subjected are mentioned in Table 2. 
Table 2. Participants' inclusion and exclusion criteria.

\begin{tabular}{|c|c|}
\hline Inclusion Criteria & Exclusion Criteria \\
\hline $\begin{array}{l}\text { Enough functional capacity to walk } \\
\text { independently }\end{array}$ & $\begin{array}{l}\text { Under medical condition that contraindicates the } \\
\text { proximity or contact with animals (exclusion criteria for } \\
\text { the intervention group) }\end{array}$ \\
\hline ASD diagnosis & $\begin{array}{l}\text { Animal or dog allergy (exclusion criteria for the } \\
\text { intervention group) }\end{array}$ \\
\hline Older than 40 years old & Dog phobia (exclusion criteria for the intervention group) \\
\hline \multirow[t]{2}{*}{$\begin{array}{l}\text { Being able to go up and down stairs } \\
\text { autonomously }\end{array}$} & $\begin{array}{l}\text { History of animal aggression (exclusion criteria for the } \\
\text { intervention group) }\end{array}$ \\
\hline & Not sign the informed consent. \\
\hline
\end{tabular}

\subsubsection{Recruitment}

The initial recruitment, screening, and baseline assessment of volunteered participants will be conducted in the two Autism federated entities of Madrid (Spain), APNA (Asociación de Padres de Niños con Autismo) and Nuevo Horizonte. The study interventions will end before 2022. The entities will offer participants to enroll in the study by explaining to patients and their legal guardians the intervention requirements. Once they express their interest, more detailed written information will be proportionated together with the informed contest which they will need to sign to finally participate.

\subsubsection{Randomization, Blinding and Confidentiality}

As has been mentioned before, the proposed study is a non-randomized controlled trial. Thus, there will be no randomization process. The allocation of the participants will be according to the criteria of the caregivers and professionals working in the associations, based on the eligibility criteria mentioned above according to the expected response to the activity in terms of applicability, tolerance, and treatment of the dog. Although participants will know their group assignment, the evaluators from the research will be blinded. Thus, each participant will be assigned an anonymous code, so that the evaluators will not know the allocation of patients. Moreover, personal information will be stored only for clinical research purposes and will be password protected to maintain confidentiality.

\subsubsection{Sample Size}

The sample size was calculated using G-Power 3.1 and based on data from GarciaVillamisar, et al. [29]. They achieved a significant improvement in the two subscales of the Tinetti Assessment Tool [29]. A total of 22 patients (11 allocated to the experimental and 11 allocated to the control group) would be needed to achieve a $97 \%$ and a $99 \%$ statistical power for the balance and gait subscales, respectively. Assuming $5 \%$ attrition, 24 patients will be recruited and allocated to one group or the other.

\subsection{Intervention}

The intervention will consist of 10 weeks and evaluations will be conducted before and after the intervention program. Both the evaluations and the intervention will be carried out in the facilities where participants live in order to avoid patient stress.

\subsubsection{Experimental Group}

The presented AAT program will compromise 20 sessions in 10 weeks. It will consist of carrying out different assisted therapeutic activities, explained below, wherein the dogs ${ }^{\prime}$ role will be to improve the physical function and ability to move around the facility when both walking and climbing stairs. In addition to this AAT intervention, the participants will also continue carrying out their daily therapeutic activities.

Participants will receive the sessions twice per week and the therapies will always be conducted at the same time. The intervention will consist of using dog-human interaction 
to walk, move through obstacle courses, climb stairs, or use a handrail. Sessions' settings will be in an environment known to the participants and with the presence of their reference professionals to facilitate their adherence and sense of security. Moreover, to have correct control of the participants, the sessions will be developed in groups of three participants. Nevertheless, if participants manifest phobia, aggression or behavioral alterations during the session that impede the correct development of the intervention and/or any reaction that may be considered dangerous for their health or the health of any of the members of the group (including the dog) they will be excluded from the experimental group.

The methodology of the sessions will be based on the facilitation of patients' movements through their dog and the natural benefits of human-dog interaction, so that, by having dogs as partners and collaborators in different activities, patients will achieve tasks that otherwise are more complicated or impossible for them. This is because people with ASD may feel pressured and not obey the instructions given by professionals. However, the presence of dogs may help to naturally conduct the desired activities and, therefore, to achieve the proposed objectives. Each session will last $30 \mathrm{~min}$. The structure of each will follow the following order. First, a welcome period of $5 \mathrm{~min}$, during which participants and technicians will introduce themselves with greetings between the patients and the dogs. Secondly, the central part of the sessions, with a duration of $20 \mathrm{~min}$, will be focused on the specific objectives of the therapy. It will consist of the execution of diverse orders given by the professional, such as performing a circuit that includes active listening, going up and downstairs, ambulation, manipulation of objects and activities that involve interaction with their companions and with the dog. The sessions will end with a farewell period of $5 \mathrm{~min}$ in order to say goodbye to the dog, sometimes with food, sometimes only verbally, gesturally, and/or with petting. This approach has been followed by previous studies [30,31].

The professional team, with a multidisciplinary profile, will be composed of AAI experts (one physiotherapist and one social educator), two psychologists, one occupational therapist, two sport science professionals and another social educator. These professionals are researchers from the University or workers at collaborating entities. The collaboration of those professionals, who daily work with the participants of this study, is crucial to adequately design the intervention, engage participants, and make them feel secure during the whole intervention. The person in charge of the dog, acting as a technician, as well as the dog itself, will be the same in all sessions for each participant.

Regarding dogs and with the aim to reduce the possibility of suffering from stress during AAI sessions [32,33], they will be selected according to their temperament, adaptability, motivation, and willingness to engage in the sessions. The handler and the dog must be pet and owner, living together, and must have a mutual strong bond to ensure the adequate interpretation of dogs' body language and avoid any kind of stress or discomfort. However, this method, wherein the owner brings their dog to the therapy, could led to transference or issues of competing priorities. While this is known, the preferred method is nonetheless handler/provider, due to the importance of identifying stress or discomfort in the dogs. In these cases, the handler will immediately stop the activity, have a break, and advance to another task. Dogs will follow a strict zoonosis procedure including behavioral, blood, urine, and feces analyses. Deworming, rabies, and tetravalent vaccinations will be also required and fulfilled. AAT Office of the Rey Juan Carlos University will be ensured animal welfare through the intervention including physical health and avoiding animal fatigue or stress presence.

To ensure animals' wellbeing, dogs will be limited to $4 \mathrm{~h} /$ week and a rest of more than 15 min between sessions when there are two consecutive sessions. Furthermore, dog-training sessions must not be more frequent than 4/day and must last less than 10 min each one. The dog must not be hungry when training and must always have access to fresh water. All these statements have been followed in other studies [34,35] will be supervised by the head and academics of the Animal-Assisted Intervention Office of the University.

The dogs who will participate in the intervention are: 
- Blues, a male Labrador Retriever, aged 5 years, sterilized and weighing $40 \mathrm{~kg}$. Blues has experience in activities with people suffering from different disabilities and older adults with cognitive impairments.

- Josi, a female mix of Labrador Retriever, aged 11 years, sterilized and weighing $22 \mathrm{~kg}$. Josi has experience with people with ASD, kids and people with drug addictions.

\subsubsection{Usual Care Group}

The control group of the study will continue with their daily life activities and the treatments carried out in their respective residences.

\subsection{Outcomes and Measures}

Participants from both groups will be evaluated at the beginning and the end of the study, within 7 days before and after the intervention starts and ends, respectively. As can be seen in Figure 1, there are three relevant times regarding evaluations: T1 (before the study begins), T2 (while the study is being doing), and T3 (once all sessions have been conducted). All variables will be evaluated twice (before and after the intervention), with the exception of the communication with the dog, which is evaluated three times during the sessions (in the first one, in the 11th and in the last one).

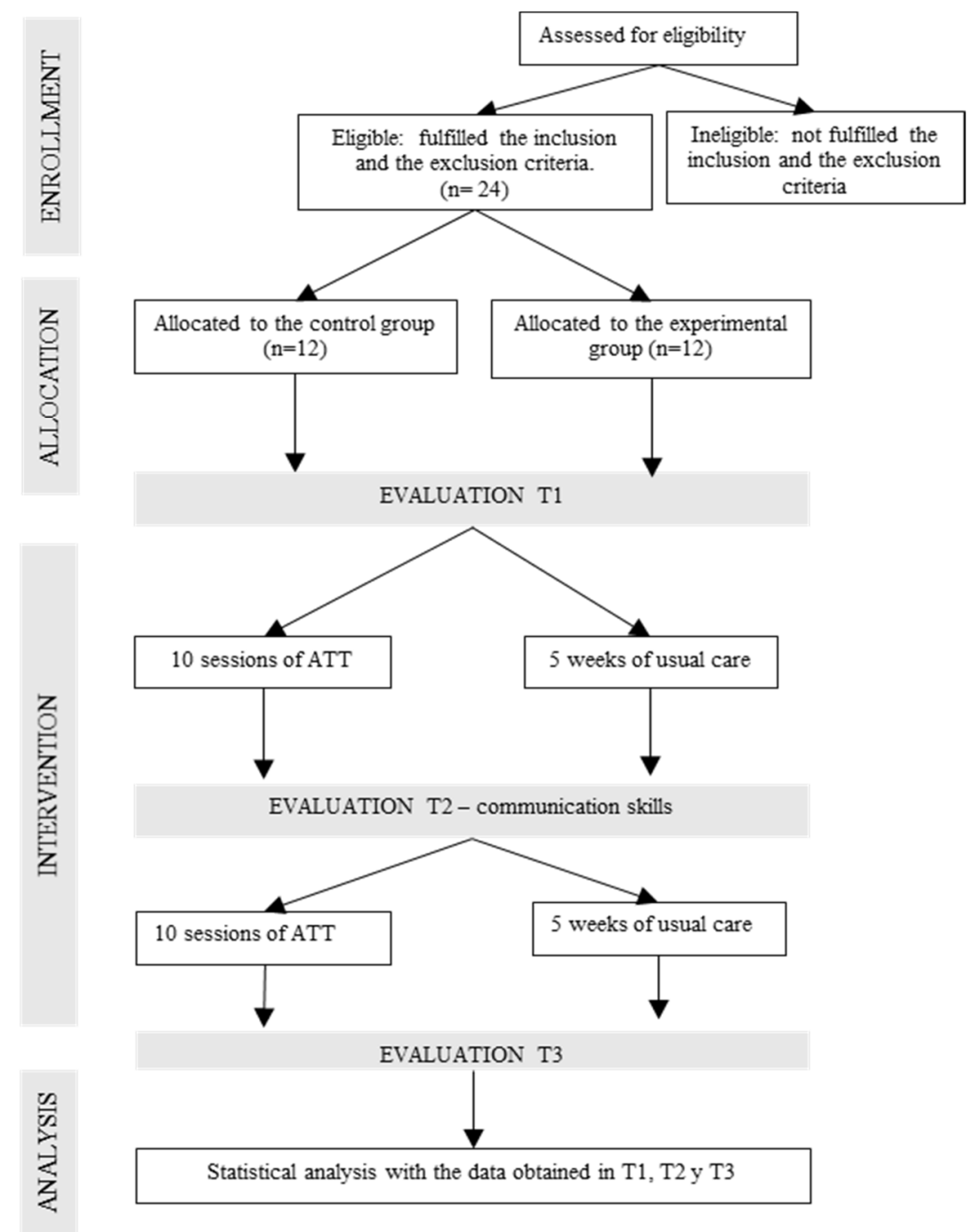

Figure 1. Protocol timeline implemented in the study.

The control group will only participate in the pre- and post-intervention evaluations, so there will be no data about communication with the dog for this group. The professionals 
who usually are in contact with the participants in each association will be in charge of carrying out the assessment tests. As can be seen in Figure 1, apart from the pre- and post-intervention evaluations (which include the assessment of communication skills) there will be an intermediate evaluation of communication skills conducted during the sessions.

First, the sociodemographic variables will be collected including age, sex, between others. Besides, anthropometric measurements (height, weight, and length of segments used for the appropriate kinematic analyses with Kinovea Software), and relevant information concerning the disability and diagnosis of each participant will be stored.

\subsubsection{Walking Performance}

The evaluation of the walking ability over 6 and $10 \mathrm{~m}$ will be carried out in a straight corridor, measuring the time spent. Participants will perform this task at their natural self-selected pace. By using a two-distance test of $6 \mathrm{~m}$ and $10 \mathrm{~m}$, in a corridor known to the participants, changes in the length of the steps, changes in cadence, and changes in the duration of the phases of the gait cycle will be evaluated. It will be performed at patients' preferred speed and in a straight line, on a smooth, non-slippery surface. To carry out this test, three marks will be placed on the ground, the starting mark, another mark at $6 \mathrm{~m}$, and another mark at $10 \mathrm{~m}$. This will allow the extraction of variables for the $6-\mathrm{m}$ and $10-\mathrm{m}$ tests. These walking tests are widely used, although the distance utilized in the literature is variable. It has been observed that a greater distance could increase the reliability of the test [36]. The 6-m test has been previously used in young adults with ASD, so obtaining data at this distance will allow comparison with that previous study [37]. The test will be recorded on video to obtain kinematic variables through the "Kinovea" software, which has shown good reliability in previous studies to obtain kinematic variables $[38,39]$.

\subsubsection{Balance and Risk of Falls}

Participants' balance and risk of falls will be assessed using the Tinetti assessment tool [40]. The scale includes a balance test and a walking test. The minimum score that could be obtained is zero points and the maximum walking score could be 12 points, whereas the maximum score for the balance component could be 16 points. So that, the total score of the scale ranges from 0 to 28 points, with scorings equal to or lower than 25 indicate the existence of a risk of falling (higher scores mean a lower risk of falls). The Tinetti scale [40] has been formerly employed to assess balance and the risk of falls in people with intellectual disabilities [41,42].

\subsubsection{Communication Abilities}

During the first, intermediate, and last session in the dogs' presence, the communication skills of the experimental group will be assessed using an adapted version of the comprehensive and expressive language scale [43] to assess interaction and communication with dogs and other people. This scale was designed by a group of experts, including researchers from different disciplines, therapists. and educators with a significant experience with people with ASD. This scale will be completed by the staff according to the frequency of the occurrence of relevant behaviors, such as staring at the dog, fixing the attention to the dog, interacting with the dog (feeding, petting, grooming ... ), speaking with other people, naming objects or people, expressing feelings, or asking questions. This scale consists of 38 items scored 2 (yes, always), 1 (sometimes) or 0 (no, never) (Table 3). The minimum score that could be reached is 0 and the maximum score 76 . Higher score scores mean better communication skills. The items are shown in Table 3. This scale will be administered during the first, intermediate and final sessions, and only in the experimental group, as the control groupwill not have contact with the dog. 
Table 3. Items observed to evaluate the patients' communication and interaction during the sessions.

\begin{tabular}{|c|c|c|}
\hline \multicolumn{3}{|c|}{ Items to Observe } \\
\hline & & \\
\hline Non-verbal communication & & Verbal communication \\
\hline $\begin{array}{l}\text { 1. Stares directly at the dog } \\
\text { 2. Briefly looks at the dog } \\
\text { 3. Focuses on the dog steadily } \\
\text { 4. Focuses on the dog intermittently } \\
\text { 5. Focuses on someone else who is } \\
\text { with the dog } \\
\text { 6. Tries to direct attention to the dog } \\
\text { 7. Draws the attention of someone or } \\
\text { 8. the dog } \\
\text { 9. Looks only at the dog, } \\
\text { 10. Looks at the person and the dog } \\
\text { 11. Objects attract his/her attention }\end{array}$ & $\begin{array}{l}\text { 12. Activities attract his/her attention } \\
\text { 13. Shows rejection towards the dog } \\
\text { 14. Touches the dog } \\
\text { 15. Feeds the dog } \\
\text { 16. Plays with the dog } \\
\text { 17. Brushes the dog } \\
\text { 18. Touches people when the dog is } \\
\text { away } \\
\text { 19. Touches people when the dog is } \\
\text { present } \\
\text { 20. Interacts voluntarily with the dog } \\
\text { 21. Uses the dog to communicate with } \\
\text { people } \\
\text { 22. Uses people to communicate with } \\
\text { the dog }\end{array}$ & $\begin{array}{ll}\text { 1. } & \text { Talks to people, } \\
\text { 2. } & \text { Talks to the dog } \\
\text { 3. } & \text { Names the dog, } \\
\text { 4. Names the people, } \\
\text { 5. Names session's objects } \\
\text { 6. Names session's activities } \\
\text { 7. Expresses or show joy } \\
\text { 8. Expresses or show sadness } \\
\text { 9. Expresses or show fear } \\
\text { 10. Expresses or shows disgust } \\
\text { 11. Expresses or shows surprise, } \\
\text { 12. } \\
\text { 13. } & \text { Requests what they want, } \\
\text { 14. } & \text { Goes to someone to ask for it } \\
\text { 15. Asks questions about the dog, } \\
\text { 16. Asks questions about people } \\
\text { 17. Answers simple questions }\end{array}$ \\
\hline
\end{tabular}

A reduced version of this scale (omitting the items related to the dog) will be administered without the presence of the dog and during the pre- and post-intervention assessments. This scale will consist of 14 items and both the control, and the experimental groups will be assessed in order to enable between-group comparisons. The minimum scoring of this 14-item scale is 0 and the maximum could be 28 . Higher scores mean better communication and interaction. Psychometric properties will be extracted of this adapted version.

\subsubsection{Ability to Climb and Descend Stairs}

Patients will be evaluated through the Climbing and Descending Stairs Test (10 steps). Climbing and descending stairs are daily activities that require coordination and good physical function and that have been used by different previous studies in people with intellectual disabilities [44,45], in addition to being validated also in other pathologies [46]. The test will consist of going up and down a flight of stairs at the preferred speed, allowing the use of the handrail if the participant so wishes. The test will be recorded on video to obtain kinematic variables through the Kinovea software, which has shown good reliability in previous studies to obtain kinematic variables [38,39].

\subsection{Statistical Analyses}

First, the Kolmogorov-Smirnov and Shapiro-Wilk tests will be used to determine whether parametric or non-parametric statistical analyses should be applied. Descriptive results will be presented as mean and SD or median (range) according to the results of the normality tests. To evaluate the effects of the program, a repeated-measures ANOVA will be used. In this ANOVA, two groups (experimental or control) and two/three-time moments (pre, intermediate, and post) will be analyzed. Additionally, a Bonferroni adjustment will be made for this analysis. Although we plan to follow the mentioned statistical process, the final enrollment and completion will determine the appropriate test to performed.

Statistical analysis will be performed using SPSS statistical software (version 26.0; SPSS, Inc., Chicago, IL, USA) and the chosen significance level will be 0.05 . All data will be analyzed and manipulated only by researchers. 


\section{Discussion}

The current study aims to improve the daily living of institutionalized adults with ASD through a dog-assisted therapy focused on the enhancement of physical function and the ability to move around corridors and stairs. The growth of scientific knowledge about ASD in terms of diagnosis and therapies, as well as the increase in life expectancy of people with this disorder, has contributed to the increase in the prevalence and incidence of this group in recent decades [47-49].This progressive increase of ASD populations is forcing the scientific, social, and health community to evaluate and optimize ASD patients' well-being together with increasing their quality of life [47-49].

Despite not having found scientific literature that could relate AAT with physical function in people with ASD, according to some studies, AAT with dogs could be a feasible complementary strategy to be included as part of the rehabilitation process experienced by people with physical disabilities, especially at the level of balance and eye-hand coordination [50]. However, the results obtained in this study, performed by Crespo et al. (2020), did not include the autistic population, and so needs to be confirmed by larger controlled clinical trials.

In this sense, the present study may represent a solid research plan to test the effectiveness of AATs in adults with ASD, in order to reduce the risk of falls through activities and circuits that involve walking and climbing or descending stairs, along with the known benefits of human-animal interaction, such as motivation, connection, calming, or stressrelieving [51,52]. Moreover, to our knowledge, this will be the first controlled trial to explore the efficacy of dog-assisted therapy on the gait of adults with ASD that may have walking disturbances due to potential physical and intellectual disturbances. Given the demonstrated importance of good psychomotor maintenance in achieving autonomy in $\mathrm{ADL}$ and quality of life, it is convenient to propose treatments that favor the development of these capacities, especially in people with neurodevelopmental disorders. AAI may be an adequate alternative to improve social interaction and communication skills since the animal acts as a social facilitator and a powerful motivator to carry out activities $[15,53,54]$, directing this technique towards psychomotor and social objectives may be a quick and effective method for enhancing the autonomy of users with ASD and improving their quality of life.

However, after conducting this study, it will be still necessary to expand the field of research with further studies, on behalf of some of the limitations of the present study. First, regarding the methodological limitations, the current study will include neither randomized nor double-blind processes, which might influence interpretations of the results. In addition, the research will be focused only on the benefits deriving from the presence of the animal, without delving into its characteristics. Second, the uncertain health conditions in Spain due to COVID-19 may change during the study, which could modify patients' behavior or intervention procedures. Furthermore, the use of a face mask may continue to be mandatory, which may affect the comfort and the communication between patients and between the patient and the staff. Moreover, due to the pandemic circumstances, this therapeutic trial has already delayed.

In spite of these limitations, the results of this study will be useful to explore a comprehensive strategy that would help patients with ASD to increase their interaction and communication skills, as well as to increase their physical function and reduce their fall risk. These potential improvements may be also accompanied by the creation of habits and affective bonds with the dog, their peers and the professionals, which may contribute to the prevention of sedentary lifestyles, isolation, and dependence, among others.

Author Contributions: Conceptualization, I.G.-C.; B.R.-M. and D.C.-M.; methodology, A.M.L.-P.; S.V., J.C.R.-R., M.M.-G., and I.G.-C.; formal analysis, A.M.L.-P.; S.V. and D.C.-M.; investigation, D.C.-M.; E.M.-N. and I.G.-G.; resources, I.G.-G.; M.M.-G., J.C.R.-R. and C.G.-P.; writing-original draft preparation, A.M.L.-P.; S.V., and I.G.-C.; writing-review and editing, A.M.L.-P. and E.M.-N.; visualization, I.G.-G.; supervision, D.C.-M. and I.G.-G.; project administration, I.G.-G. All authors have read and agreed to the published version of the manuscript. 
Funding: The current study is funded by Rey Juan Carlos University through the Animal Assisted Intervention Office. The author A.M.L.-P. is supported by the Industrial Doctorate Spanish National grant program, part of the Strategic Plan on Science and Innovation Support of the Spanish Ministry of Science, Innovation, and Universities. The predoctoral industry grant identification number is DIN2018- 010129.

Institutional Review Board Statement: The study will be conducted according to the guidelines of the Declaration of Helsinki and approved by the Ethics Committee of Rey Juan Carlos University (approval number: 1901202103121).

Informed Consent Statement: Informed consent was obtained from all subjects involved in the study.

Acknowledgments: The authors acknowledge the support provided by Paloma Esteban Fernández, from the "Nuevo Horizonte" association, Javier Vallejo Bermejo, from Animal Nature, Sergio Soriano Carceller, from APNA, and Luis Lucio Lobato-Rincón and Cristina Martín Sánchez from the Animalassisted intervention office of the Rey Juan Carlos University.

Conflicts of Interest: The authors declare no conflict of interest. Moreover, the funders had no role in the design of the study; in the collection, analyses, or interpretation of data; in the writing of the manuscript, or in the decision to publish the results.

\section{References}

1. Association, A.P. Guía de Consulta de Los Criterios Diagnósticos del DSM- $5^{\circledR}$ : Spanish Edition of the Desk Reference to the Diagnostic Criteria From DSM- $5^{\circledR}$; American Psychiatric Publishing: Arlington, TX, USA, 2014.

2. Pina-Camacho, L.; Villero, S.; Fraguas, D.; Boada, L.; Janssen, J.; Navas-Sánchez, F.J.; Mayoral, M.; Llorente, C.; Arango, C.; Parellada, M. Autism spectrum disorder: Does neuroimaging support the DSM-5 proposal for a symptom dyad? A systematic review of functional magnetic resonance imaging and diffusion tensor imaging studies. J. Autism Dev. Disord. 2012, 42, $1326-1341$. [CrossRef]

3. Armitano, C.; Bennett, H.; Haegele, J.; Morrison, S. Assessment of the gait-related acceleration patterns in adults with autism spectrum disorder. Gait Posture 2020, 75, 155-162. [CrossRef]

4. Lum, J.A.; Shandley, K.; Albein-Urios, N.; Kirkovski, M.; Papadopoulos, N.; Wilson, R.B.; Enticott, P.G.; Rinehart, N.J. MetaAnalysis Reveals Gait Anomalies in Autism. Autism Res. 2021, 14, 733-747. [CrossRef] [PubMed]

5. Zúñiga, A.H.; Balmaña, N.; Salgado, M. Los trastornos del espectro autista (TEA). Pediatr. Integral 2017, $21,92-108$.

6. Hume, K.; Steinbrenner, J.R.; Odom, S.L.; Morin, K.L.; Nowell, S.W.; Tomaszewski, B.; Szendrey, S.; McIntyre, N.S.; YücesoyÖzkan, S.; Savage, M.N. Evidence-based practices for children, youth, and young adults with autism: Third generation review. J. Autism Dev. Disord. 2021, 51, 4013-4032. [CrossRef] [PubMed]

7. Odom, S.L.; Hall, L.J.; Morin, K.L.; Kraemer, B.R.; Hume, K.A.; McIntyre, N.S.; Nowell, S.W.; Steinbrenner, J.R.; Tomaszewski, B.; Sam, A.M. Educational Interventions for Children and Youth with Autism: A 40-Year Perspective. J. Autism Dev. Disord. 2021, 1-16. [CrossRef] [PubMed]

8. Jegatheesan, B.; Beetz, A.; Ormerod, E.; Johnson, R.; Fine, A.; Yamazaki, K.; Dudzik, C.; Garcia, R.; Winkle, M.; Choi, G. IAHAIO Whitepaper 2014 (updated for 2018). The IAHAIO Definitions for Animal Assisted Intervention and Guidelines for Wellnes of Animals Involved in AAI. Available online: http:/ /iahaio.org/best-practice/white-paper-on-animal-assisted-interventions / (accessed on 11 April 2018).

9. Fine, A.H. Handbook on Animal-Assisted Therapy: Foundations and Guidelines for Animal-Assisted Interventions; Academic Press: Cambridge, MA, USA, 2019.

10. Hart, L.A. Positive Effects of Animals for Psychosocially Vulnerable People: A Turning Point for Delivery. In Handbook on Animal-Assisted Therapy; Elsevier: Amsterdam, The Netherlands, 2010; pp. 59-84.

11. O'Haire, M.E. Animal-assisted intervention for autism spectrum disorder: A systematic literature review. J. Autism Dev. Disord. 2013, 43, 1606-1622. [CrossRef] [PubMed]

12. Wijker, C.; Leontjevas, R.; Spek, A.; Enders-Slegers, M.-J. Process Evaluation of Animal-Assisted Therapy: Feasibility and Relevance of a Dog-Assisted Therapy Program in Adults with Autism Spectrum Disorder. Animals 2019, 9, 1103. [CrossRef] [PubMed]

13. Yap, E.; Scheinberg, A.; Williams, K. Attitudes to and beliefs about animal assisted therapy for children with disabilities. Complementary Ther. Clin. Pract. 2017, 26, 47-52. [CrossRef] [PubMed]

14. Ghai, J.L.; Lehr, D.H.; Harayama, N.E.; Spooner, F.; Protopopova, A. Animal-assisted Interventions as an Adjunct to ABA Services: Clinician Practices and Perceptions. Behav. Anal. Pract. 2021. [CrossRef]

15. Wijker, C.; Leontjevas, R.; Spek, A.; Enders-Slegers, M.-J. Effects of dog assisted therapy for adults with autism spectrum disorder: An exploratory randomized controlled trial. J. Autism Dev. Disord. 2020, 50, 2153-2163. [CrossRef] 
16. Ávila-Álvarez, A.; Alonso-Bidegain, M.; De-Rosende-Celeiro, I.; Vizcaíno-Cela, M.; Larrañeta-Alcalde, L.; Torres-Tobío, G. Improving social participation of children with autism spectrum disorder: Pilot testing of an early animal-assisted intervention in Spain. Health Soc. Care Community 2020, 28, 1220-1229. [CrossRef] [PubMed]

17. Ben-Itzchak, E.; Zachor, D.A. Dog training intervention improves adaptive social communication skills in young children with autism spectrum disorder: A controlled crossover study. Autism Int. J. Res. Pract. 2021, 25, 1682-1693. [CrossRef] [PubMed]

18. Germone, M.M.; Gabriels, R.L.; Guérin, N.A.; Pan, Z.; Banks, T.; O'aire, M.E. Animal-assisted activity improves social behaviors in psychiatrically hospitalized youth with autism. Autism Int. J. Res. Pract. 2019, 23, 1740-1751. [CrossRef]

19. Griffioen, R.E.; van der Steen, S.; Verheggen, T.; Enders-Slegers, M.J.; Cox, R. Changes in behavioural synchrony during dogassisted therapy for children with autism spectrum disorder and children with Down syndrome. J. Appl. Res. Intellect. Disabil. JARID 2020, 33, 398-408. [CrossRef]

20. Silva, K.; Correia, R.; Lima, M.; Magalhães, A.; de Sousa, L. Can dogs prime autistic children for therapy? Evidence from a single case study. J. Altern. Complementary Med. 2011, 17, 655-659. [CrossRef]

21. Leung, J.Y.; Mackenzie, L.; Dickson, C. Outcomes of assistance dog placement in the home for individuals with autism spectrum disorder and their families: A pilot study. Aust. Occup. Ther. J. 2021. [CrossRef]

22. Hill, J.; Ziviani, J.; Driscoll, C.; Teoh, A.L.; Chua, J.M.; Cawdell-Smith, J. Canine Assisted Occupational Therapy for Children on the Autism Spectrum: A Pilot Randomised Control Trial. J. Autism Dev. Disord. 2020, 50, 4106-4120. [CrossRef]

23. Uccheddu, S.; Albertini, M.; Pierantoni, L.; Fantino, S.; Pirrone, F. The Impacts of a Reading-to-Dog Programme on Attending and Reading of Nine Children with Autism Spectrum Disorders. Anim. Open Access J. MDPI 2019, 9, 491. [CrossRef] [PubMed]

24. Silva, K.; Lima, M.; Santos-Magalhães, A.; Fafiães, C.; de Sousa, L. Can Dogs Assist Children with Severe Autism Spectrum Disorder in Complying with Challenging Demands? An Exploratory Experiment with a Live and a Robotic Dog. J. Altern. Complementary Med. 2018, 24, 238-242. [CrossRef] [PubMed]

25. Wijker, C.; Kupper, N.; Leontjevas, R.; Spek, A.; Enders-Slegers, M.J. The effects of Animal Assisted Therapy on autonomic and endocrine activity in adults with autism spectrum disorder: A randomized controlled trial. Gen. Hosp. Psychiatry 2021, 72, 36-44. [CrossRef] [PubMed]

26. Viau, R.; Arsenault-Lapierre, G.; Fecteau, S.; Champagne, N.; Walker, C.D.; Lupien, S. Effect of service dogs on salivary cortisol secretion in autistic children. Psychoneuroendocrinology 2010, 35, 1187-1193. [CrossRef]

27. Covarrubias-Pineda, E.-S. Eficacia de la Terapia Asistida con Animales en Personas con un Diagnóstico de Trastorno del Espectro Autista. Revisión Sistemática; Universidad de Salamanca: Salamanca, Spain, 2019.

28. Chan, A.-W.; Tetzlaff, J.M.; Altman, D.G.; Laupacis, A.; Gøtzsche, P.C.; Krleža-Jerić, K.; Hróbjartsson, A.; Mann, H.; Dickersin, K.; Berlin, J.A. SPIRIT 2013 statement: Defining standard protocol items for clinical trials. Ann. Intern. Med. 2013, 158, 200-207. [CrossRef] [PubMed]

29. Garcia-Villamisar, D.; Dattilo, J.; Muela, C. Effects of B-active2 on balance, gait, stress, and well-being of adults with autism spectrum disorders and intellectual disability: A controlled trial. Adapt. Phys. Act. Q. 2017, 34, 125-140. [CrossRef]

30. Quintavalla, F.; Cao, S.; Spinelli, D.; Caffarra, P.; Rossi, F.M.; Basini, G.; Sabbioni, A. Effects of Dog-Assisted Therapies on Cognitive Mnemonic Capabilities in People Affected by Alzheimer's Disease. Animals 2021, 11, 1366. [CrossRef] [PubMed]

31. Menna, L.F.; Santaniello, A.; Amato, A.; Ceparano, G.; Di Maggio, A.; Sansone, M.; Formisano, P.; Cimmino, I.; Perruolo, G.; Fioretti, A. Changes of Oxytocin and Serotonin Values in Dialysis Patients after Animal Assisted Activities (AAAs) with a Dog-A Preliminary Study. Animals 2019, 9, 526.

32. Haubenhofer, D.K.; Kirchengast, S. Physiological arousal for companion dogs working with their owners in animal-assisted activities and animal-assisted therapy. J. Appl. Anim. Welf. Sci. 2006, 9, 165-172. [CrossRef] [PubMed]

33. Haubenhofer, D.K.; Kirchengast, S. 'Dog handlers' and dogs' emotional and cortisol secretion responses associated with animalaassisted therapy sessions. Soc. Anim. 2007, 15, 127-150. [CrossRef]

34. Lobato Rincón, L.L.; Rivera Martín, B.; Medina Sánchez, M.Á.; Villafaina, S.; Merellano-Navarro, E.; Collado-Mateo, D. Effects of dog-assisted education on physical and communicative skills in children with severe and multiple disabilities: A pilot study. Animals 2021, 11, 1741. [CrossRef] [PubMed]

35. Lavín-Pérez, A.M.; Martín-Sánchez, C.; Martínez-Núñez, B.; Lobato-Rincón, L.L.; Villafaina, S.; González-García, I.; Mata-Cantero, A.; Graell, M.; Merellano-Navarro, E.; Collado-Mateo, D. Effects of dog-assisted therapy in adolescents with eating disorders: A study protocol for a pilot controlled trial. Animals 2021, 11, 2784. [CrossRef]

36. Peters, D.M.; Fritz, S.L.; Krotish, D.E. Assessing the reliability and validity of a shorter walk test compared with the 10-Meter Walk Test for measurements of gait speed in healthy, older adults. J. Geriatr. Phys. Ther. 2013, 36, 24-30. [CrossRef]

37. Weiss, M.J.; Moran, M.F.; Parker, M.E.; Foley, J.T. Gait analysis of teenagers and young adults diagnosed with autism and severe verbal communication disorders. Front. Integr. Neurosci. 2013, 7, 33. [CrossRef]

38. Fernández-González, P.; Koutsou, A.; Cuesta-Gómez, A.; Carratalá-Tejada, M.; Miangolarra-Page, J.C.; Molina-Rueda, F. Reliability of Kinovea ${ }^{\circledR}$ Software and Agreement with a Three-Dimensional Motion System for Gait Analysis in Healthy Subjects. Sensors 2020, 20, 3154. [CrossRef] [PubMed]

39. Puig-Diví, A.; Escalona-Marfil, C.; Padullés-Riu, J.M.; Busquets, A.; Padullés-Chando, X.; Marcos-Ruiz, D. Validity and reliability of the Kinovea program in obtaining angles and distances using coordinates in 4 perspectives. PLoS ONE 2019, 14, e0216448. [CrossRef] [PubMed] 
40. Tinetti, M.E.; Williams, T.F.; Mayewski, R. Fall risk index for elderly patients based on number of chronic disabilities. Am. J. Med. 1986, 80, 429-434. [CrossRef]

41. Chiba, Y.; Shimada, A.; Yoshida, F.; Keino, H.; Hasegawa, M.; Ikari, H.; Miyake, S.; Hosokawa, M. Risk of fall for individuals with intellectual disability. Am. J. Intellect. Dev. Disabil. 2009, 114, 225-236. [CrossRef] [PubMed]

42. Aldao, D.M.; Lemos, R.M.; Vázquez, S.P.; Pérez, C.A. Efecto de un programa de ejercicio físico sobre el riesgo de caídas, equilibrio y velocidad de la marcha en personas mayores con discapacidad intelectual. Rehabilitacion 2020, 54, 19-24. [CrossRef] [PubMed]

43. Rodríguez Santos, F. Evaluación del lenguaje: Elaboración de un sistema de análisis del lenguaje comprensivo y expresivo. Ph.D. Thesis, Universidad Autónoma de Madrid, Madrid, Spain, 1993.

44. Carmeli, E.; Imam, B.; Merrick, J. The relationship of pre-sarcopenia (low muscle mass) and sarcopenia (loss of muscle strength) with functional decline in individuals with intellectual disability (ID). Arch. Gerontol. Geriatr. 2012, 55, 181-185. [CrossRef]

45. Shieh, W.-Y.; Ju, Y.-Y.; Yu, Y.-C.; Lin, C.-K.; Lin, Y.-T.; Cheng, H.-Y.K. Stair-walking performance in adolescents with intellectual disabilities. Sensors 2016, 16, 1066. [CrossRef]

46. Collado-Mateo, D.; Domínguez-Muñoz, F.J.; Olivares, P.R.; Adsuar, J.C.; Gusi, N. Stair negotiation in women with fibromyalgia: A descriptive correlational study. Medicine 2017, 96, e8364. [CrossRef]

47. Data \& Statistics on Autism Spectrum Disorder I CDC. Centers for Disease Control and Prevention. 2019. Available online: https:/ / www.cdc.gov/ncbddd/autism/data.html (accessed on 13 February 2021).

48. Zablotsky, B.; Black, L.I.; Maenner, M.J.; Schieve, L.A.; Blumberg, S.J. Estimated prevalence of autism and other developmental disabilities following questionnaire changes in the 2014 National Health Interview Survey. Natl. Health Stat. Rep. 2015, 87, 1-20.

49. André, T.G.; Montero, C.V.; Félix, R.E.O.; Medina, M.E.G. Prevalencia del trastorno del espectro autista: Una revisión de la literatura. Jóvenes Cienc. 2020, 7, 1-7.

50. Crespo, A.A.; Lemos, R.I.M.; Pérez, C.A. Factibilidad de la terapia asistida con perros como estrategia para mejorar el equilibrio y la motricidad en personas con discapacidad física. E-Motion Rev. De Educ. Mot. E Investig. 2020, 14, 15-29.

51. Hunt, M.G.; Chizkov, R.R. Are therapy dogs like Xanax? Does animal-assisted therapy impact processes relevant to cognitive behavioral psychotherapy? Anthrozoös 2014, 27, 457-469. [CrossRef]

52. Shen, R.Z.Z.; Xiong, P.; Chou, U.I.; Hall, B.J. "We need them as much as they need us": A systematic review of the qualitative evidence for possible mechanisms of effectiveness of animal-assisted intervention (AAI). Complementary Ther. Med. 2018, 41, 203-207. [CrossRef] [PubMed]

53. Dimolareva, M.; Dunn, T.J. Animal-Assisted Interventions for School-Aged Children with Autism Spectrum Disorder: A Meta-Analysis. J. Autism Dev. Disord. 2020, 51, 2436-2449. [CrossRef] [PubMed]

54. Zhao, M.; Chen, S.; You, Y.; Wang, Y.; Zhang, Y. Effects of a Therapeutic Horseback Riding Program on Social Interaction and Communication in Children with Autism. Int. J. Environ. Res. Public Health 2021, 18, 2656. [CrossRef] 